\title{
Independence Between Two Channels of Surface Electromyogram Signal to Measure the Loss of Motor Units
}

\author{
Sridhar P. Arjunan ${ }^{1}$, Dinesh Kumar ${ }^{1}$, Ganesh Naik ${ }^{2}$ \\ ${ }^{1}$ Bio-signals Lab, School of Electrical and Computer Engineering, RMIT University, \\ GPO Box 2476, Melbourne, Victoria 3001, Australia, sridhar.arjunan@rmit.edu.au \\ ${ }^{2}$ Faculty of Engineering and Information Technology (FEIT), University of Technology Sydney (UTS), \\ Sydney - 2007, Australia.
}

\begin{abstract}
This study has investigated the relationship in the connectivity of motor units in surface electromyogram (sEMG) of biceps brachii muscle. It is hypothesized that with ageing, there is reduction/loss in number of motor units, leading to reduction in the independence between the channels of the recorded muscle activity. Two channels of sEMG were recorded during three levels of isometric muscle contraction: $50 \%, 75 \%$ and $100 \%$ maximal voluntary contraction (MVC). 73 subjects (age range 20-70) participated in the experiments. The independence in channel index (ICI) between the two sEMG recording locations was computed using the independent components and Frobenius norm. ANOVA Statistical analysis was performed to test the effect of age (loss of motor units) and level of contraction on ICI. The results show that the ICI among the older cohort was significantly lower compared with the younger adults. This research study has shown that the reduction in number of motor units is reflected by the reduction in the ICI of the SEMG signal.
\end{abstract}

Keywords: Independence, motor units, surface electromyogram, control.

\section{INTRODUCTION}

$\mathrm{T}$ HERE IS AGE associated reduction in strength and precision, with older adults having a reduced ability to maintain a steady force [1], [2]. Neuromuscular changes associated with ageing lead to reduction in active motor neurons leading to an increase in the innervations ratio of low-threshold motor units among the elderly [3], [4]. The predominant loss of type II fibers in the muscle [5], [6] is also accompanied with remodeling of the motor units of the muscle and with increase in motor unit (MU) size due to age [7], [8]. While there is reduction in type II muscle fibers and motor units [9], [10], there is compensatory increase in the number of type I fibers. The functional significance of age associated increase in the size of low-threshold motor units is reduction in the range of motor recruitment, leading to reduced muscle control [9], even when the muscle strength or endurance may largely be unchanged [10].

The effect of change in size and number of motor units on motor unit recruitment due to age has been reported [11]. However, earlier studies have not reported on age-associated significant increase in the synchronization of multiple channels. We hypothesize that there would not be any increase in synchronization but there would be reduction in independence between the multiple channels because of the statistically random nature of motor unit recruitment. Independence between channels index (ICI) of surface electromyogram (sEMG) is a measure of the independence between multiple surface recordings of the muscle activation.

Reduction in number of motor units leads to an overall reduction in the independency between the recorded sEMG channels. This study aims to investigate the effect of age on the independence between multiple channels by computing ICI of the two surface recordings of the muscle activity.
We hypothesize that there will be age associated reduction in independence between multiple channels recorded from a muscle. Thus, the older cohort will have a lower ICI compared with younger people. This study reports testing of the above hypothesis by measuring the ICI of the young and the older cohort during a short duration isometric muscle contraction.

\section{SUBJECT \& METHODS}

\subsection{Human experiments ethics clearance}

Prior to the start of the experiment, the purpose of the study, procedures and risks associated with participation were explained to each subject. A plain language statement outlining the details of the experiments was provided to each participant, and written consent was obtained. The experiments were approved by RMIT University Human Research Ethics Committee and were conducted in accordance with Declaration of Helsinki 1975, as revised in 2004.

\subsection{Subjects}

Seventy three healthy participants were recruited for this study. The exclusion criteria were: (i) any history of neuromuscular disorder, (ii) recent injury to the right upperlimb, and (iii) left-hand dominance. The subjects were divided into five groups based on their age as mentioned below:

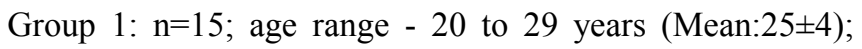
Group 2: $\mathrm{n}=15$; age range -30 to 39 years (Mean:35 \pm 3.9 ); Group 3: $n=15$; age range -40 to 49 years (Mean:43 \pm 4.9 ) ; Group 4: $n=15$; age range -50 to 59 years (Mean:55 \pm 4.6 ); Group 5: $n=13$; age range -60 to 69 years (Mean:65 \pm 3.05 ). 


\subsection{EMG recording procedures}

Surface EMG signal was recorded using Delsys (Boston, MA, USA), a proprietary sEMG acquisition system. The sampling rate used was 1000 samples per second. Delsys bipolar electrodes (Boston, MA, USA) were used in this study. These are active electrodes with two silver bars mounted directly on the preamplifier with a fixed interelectrode distance of $10 \mathrm{~mm}$.

Two bipolar electrode channels were placed on the anterior of the arm above the biceps in a line between the antecubital fossa (lateral to the biceps brachii tendon) and the acromion process, $1 / 3$ of the distance from the antecubital fossa. Reference electrodes were placed on a bony prominence at the elbow. Prior to electrode placement, the skin was cleaned with alcohol swabs and lightly abraded with a paper towel to reduce skin impedance and ensure good adhesion of the electrodes.

\subsection{Experimental protocol}

During the experiments, the volunteers were seated on a purpose-built chair, where the feet of the subject are flat on the floor, the upper right arm is resting on the surface of an adjustable desk and the forearm is vertical, with the elbow maintained at 90 degrees. A wall-mounted force sensor (Stype force sensor - INTERFACE SM25) was attached to a wrist bracelet with a flexible steel wire. The height of the sensor was adjusted such that the cable was horizontal. The output of the force sensor was recorded and displayed in real time on the screen to the user.

\section{a) MVC calculation}

At the start of the experiment, the maximal voluntary contraction (MVC) was determined by taking the maximum of 3 MVCs, each contraction of 5 seconds duration and performed with 120 seconds rest-time between each effort. The experiment was repeated if there were any outliers with difference greater than $10 \%$.

\section{b) Isometric experiment}

After determining MVC, the participants performed three isometric contractions: at $50 \%, 75 \%$ and $100 \%$ MVCs, respectively. The participants were provided with visual feedback of the force of contraction to assist them in maintaining constant force. Each contraction was for 5 seconds duration, and a 60 minutes rest period was given between each level of contraction for recovery. Surface EMG and the force of contraction were recorded during the experiment.

\subsection{Data analysis}

\section{a) Independence between channels index (ICI)}

Independence between channels index (ICI) was computed after discarding the initial and the final one second of the recordings of sEMG and force to avoid the initial force stabilization and end of the force production. The method has been summarized as below:

Step 1: After discarding the initial and final one second due to the force stabilization, the signal recordings of both channels were passed through a set of four filters: one low pass with the approximate $3 \mathrm{~dB}$ cutoff frequencies being at 125 and three 4th order Butterworth band-pass filters with approximate $3 \mathrm{~dB}$ cutoff frequencies at $125-250 \mathrm{~Hz}, 250$ $375 \mathrm{~Hz}$ and $375-500 \mathrm{~Hz}$ (Fig.1.). This resulted in four subcomponents for each channel.

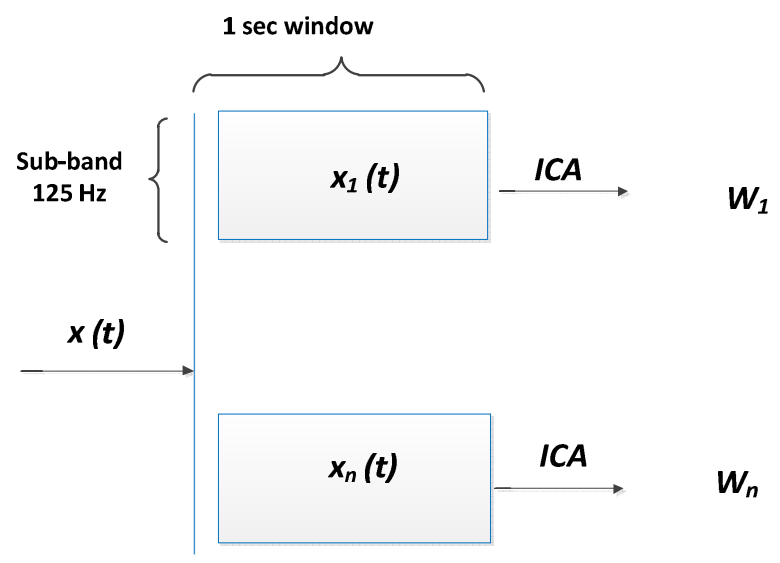

Fig.1. Representation of the data analysis.

Step 2: The next step was the estimation of un-mixing matrices for the two sets of recordings, repeated for each of the sub-bands using Fast ICA [12], [13].

$$
\mathbf{G}_{\mathrm{i}}=\mathbf{W}_{\mathrm{i}} \times \mathbf{W}_{\mathrm{i}+1}^{-1}
$$

where $\mathbf{W}_{\mathrm{i}}$ and $\mathbf{W}_{\mathrm{i}+1}^{-1} \quad$ (for $\mathrm{i}=1, . ., 3$ ) are the un-mixing matrices of spectrally adjoining windows. The global matrix was obtained by selecting the Gl that corresponded to the minimum sum of errors, which is the sum of all cells in the matrix after removing the dominant cells from every row.

Step 3: The Frobenius norm of $\mathrm{G}$ was computed (2) to obtain $\|\mathrm{G}\|$.

$$
\|\mathbf{G}\|=\sqrt{\sum_{i=1}^{N} \sum_{j=1}^{N}\left|g_{i j}\right|^{2}}
$$

where $\mathrm{g}_{\mathrm{ij}}$ is the element of $\mathbf{G}$.

Average of $\|\mathrm{G}\|$ of all the time windows in the segment was computed to obtain $\overline{\|G\|}$, and ICI index corresponding to the segment was obtained by computing, the normalized determinant of the global matrix $\log \overline{\| \mathrm{G}} \|$ (for more details, refer our previous reported study [12]). 


\section{b) Statistical Analysis}

A 5 group, 3 way $(5 \times 3)$ ANOVA was modeled in MATLAB environment for determining the statistical significance of the result. 'Age' (groups 1 to 5) and 'Force levels' ( $100 \%, 75 \%$ and $50 \%$ MVC) were the factors used to test the significance of the ICI index of sEMG.

\section{RESULTS}

Fig.2. shows the plot of the ICI for the five age groups. From this figure, it is observed that older adults have lower ICI compared with the young adults for all force levels. It is also observed that the decrease in ICI for the older cohort is high at higher force levels when compared with the younger cohort. This is due to the change in the recruitment pattern of motor units at higher force levels. We can observe this in the ICI significantly for the older cohort due to the reduction/loss of motor units as we age.

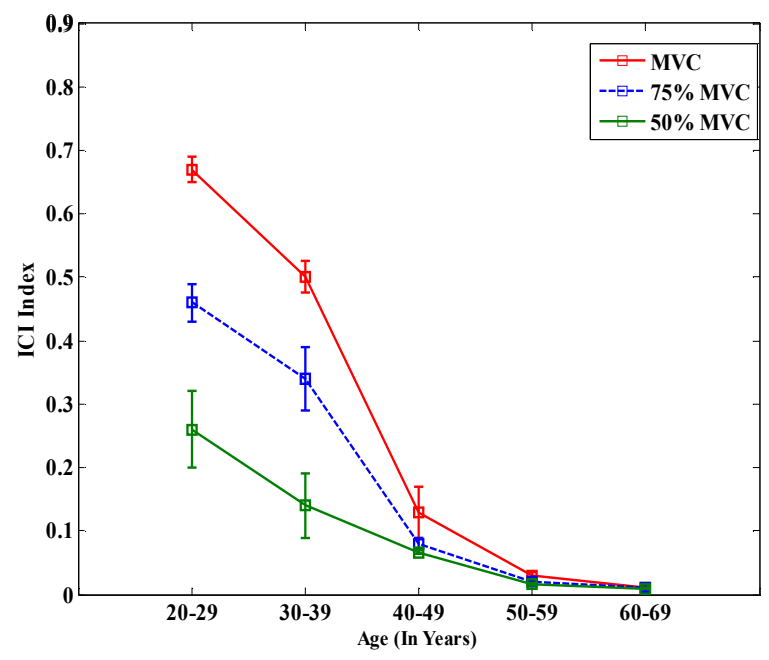

Fig.2. Independence in Channel Index (ICI) for all age groups at $100 \%, 75 \%$ and $50 \%$ MVC.

ANOVA statistical model was generated in MATLAB to observe the effect of 'Age' and 'Force levels' on the ICI measure. It is observed from the statistical analysis that ICI has significant effect due to the 'Age' groups (20-29, 30-39, $40-49,50-59,60-69$ years $)$ analysis $[\mathrm{F}(4,219)=135, \mathrm{p}=0.02$ $(p<0.05)]$. Similarly, ICI has no significant effect due to the various levels of contraction ('Force level') when combined for all age groups $[\mathrm{F}(2,282)=20.84, p=0.19]$.

\section{DISCUSSION}

The results confirm the hypothesis that there is age associated reduction in the ICI index. This decrease is indicative of reduction in the independency between sEMG recorded from the two locations in the muscle, which is caused by reduced number of motor units. It has to be noted that independence between different MUs is not similar to the synchronization between them.

Research by Semmeler et al. [14] has shown that there is no age associated change in the synchronization of different motor units in the biceps. Synchronization requires the events causing the signals to occur at the same time, or in unison, which requires the sources of the two signals to be the same or connected. However, dependence between two channels is indicative of the stochastic relationship between the two signals that may be caused by reduced number or increased size of each unit.

While synchronization between the different recordings in the muscle would indicate the functional relations within the human central nervous system (CNS) and their role in the performance of voluntary contractions [14], the dependence between the channels is associated with reduced independence between the different sEMG channels and can be caused by reduced number of motor units.

This research study has shown that with ageing, the reduction in the number of motor units is reflected by the reduction in the ICI. This study confirms that there is age associated reduction in independence between multiple channels in a muscle, and can be considered as a noninvasive method for monitoring age-associated changes of the neuromuscular properties in muscle activation.

\section{CONCLUSION}

In this study we have computed and tested the independence in motor units (ICI) to measure the age related changes in the loss of motor units and muscle activation. We have summarized the following points resulting from this study:

- Older adults have significantly lower value of the ICI index measure compared with young adults.

- There is a clear trend of decrease in the ICI measure for 20-39 age groups with the 50-69 age groups, with 40-49 age groups acting as clear threshold measure in between them.

- One of the notable arguments is in terms of step size of the ICI index which is more for the old age group than for young adults.

- With ageing, there is a reduction in the number of motor units, which would affect the normal recruitment pattern of the motor units to produce muscle contraction. This reduction in the number of motor units can result in a smaller number of connectivity between the motor units during a muscle contraction. This would in turn indicate that there is a reduction in the independence between multiple channels recorded during an isometric muscle activity.

- The index, ICI, will be useful in measuring and monitoring the age related changes in the neuromuscular activity.

\section{REFERENCES}

[1] Galganski, M.E., Fuglevand, A.J., Enoka, R.M. (1993). Reduced control of motor output in a human hand muscle of elderly subjects during submaximal 
contractions. Journal of Neurophysiology, 69, 2108 2115.

[2] Laidlaw, D.H., Bilodeau, M., Enoka, R.M. (1997). Motor unit discharge is more variable in older adults performing slow finger movements. Medicine \& Science in Sports \& Exercise, 29, 22.

[3] Kadhiresan, V.A., Hassett, C.A., Faulkner, J.A. (1996). Properties of single motor units in medial gastrocnemius muscles of adult and old rats. Journal of Physiology, 493, 543-552.

[4] Kanda, K., Hashizume, K. (1989). Changing in properties of the medial gastrocnemius motor units in aging rats. Journal of Neurophysiology, 61, 737-746.

[5] Frontera, W.R., Hughes, V.A., Fielding, R.A. (2000). Aging of skeletal muscle: A 12-yr longitudinal study. Journal of Applied Physiology, 88, 1321-1326.

[6] Evans, W.J., Lexell, J. (1995). Human aging, muscle mass and fiber type composition. The Journals of Gerontology A, 50A (Special Issue), 11-16.

[7] Andersen, J.L., Aagaard, P. (2010). Effects of strength training on muscle fiber types and size; consequences for athletes training for high-intensity sport. Scandinavian Journal of Medicine \& Science in Sports, 20 (Suppl 2), 32-38.

[8] Larsson, L., Ansved, T. (1995). Effects of ageing on the motor unit. Progress in Neurobiology, 45, 397458 .
[9] Rogers, M.A., Evans, W.J. (1993). Changes in skeletal muscle with aging, effects of exercise training. Exercise and Sport Sciences Reviews, 25, 65-102.

[10] Porter, M.M., Vandervoort, A.A., Lexel, J. (1995). Aging of human muscle: Structure, function and adaptability. Scandinavian Journal of Medicine \& Science in Sports, 5, 129-142.

[11] Erim, Z., Faisal Beg, M., Burke, D.T., de Luca, C.J. (1999). Effects of aging on motor-unit control properties. Journal of Neurophysiology, 82 (5), 20812091.

[12] Kumar, D.K., Arjunan, S.P., Naik, G.R. (2011). Measuring Increase in synchronisation to identify muscle endurance limit. IEEE Transactions on Neural Systems and Rehabilitation Engineering, 19 (5), 578587.

[13] Naik, G.R., Kumar, D.K., Arjunan, S.P. (2010). Pattern classification of Myo-Electrical signal during different Maximum Voluntary Contractions: A study using BSS techniques. Measurement Science Review, 10 (1), 1-6.

[14] Semmler, J.G. (2002). Motor unit synchronization and neuromuscular performance. Exercise and Sport Sciences Reviews, 30 (1), 8-14.

Received October 20, 2014. Accepted June 24, 2015. 